SHS-96-4

UUITP-08/96

25 March 1996

\title{
Path Integrals and Parastatistics
}

\author{
Alexios P. Polychronakos ${ }^{\dagger}$ \\ Centre for Advanced Study, Norwegian Academy of Science and Letters, \\ 0205 Oslo, Norway \\ and \\ Theoretical Physics Dept., Uppsala University \\ S-751 08 Uppsala, Sweden *
}

\begin{abstract}
The propagator and corresponding path integral for a system of identical particles obeying parastatistics are derived. It is found that the statistical weights of topological sectors of the path integral for parafermions and parabosons are simply related through multiplication by the parity of the permutation of the final positions of the particles. Appropriate generalizations of statistics are proposed obeying unitarity and factorizability (strong cluster decomposition). The realization of simple maximal occupancy (Gentile) statistics is shown to require ghost states.
\end{abstract}

$\dagger$ poly@calypso.teorfys.uu.se

* Permanent address 
Generalizations of the standard concepts of fermions and bosons have been extensively considered in the last few decades. These can be broadly divided into "phenomenological" (anyons [1-4], exclusion statistics [5]), which are meant to give a better description or understanding of systems of otherwise ordinary fermions or bosons, and "fundamental," which would be genuinely new kinds of particles. A nice review of these approaches can be found in [6], and a short and concise resumé of some relevant results can be found in [7].

The first ever consistent extension of fundamental statistics, given by Green [8], is parastatistics. In that, the standard bosonic or fermionic fields which would create identical particles are replaced by composite fields whose components commute with themselves and anticommute with each other for parabosons, or vice versa for parafermions. The number of components of the fields $p$ defines the "order" of parastatistics. In general, one can put at most $p$ parafermions in a totally symmetric wavefunction, and at most $p$ parabosons in a totally antisymmetric one. The degeneracies of occupation of more general multiparticle states are in principle calculable but rather complicated. Parastatistics in this approach has been well-studied [9-12].

The above is a field theoretic realization of parastatistics. Just as in the case of fermions or bosons, one can deal with a parastatistical system at a fixed particle number in a first-quantized formalism. In this approach, due mainly to Messiah and Greenberg $[13,14]$, the $N$-body Hilbert space is decomposed into irreducible representations (irreps) of the particle permutation group $S_{N}$. Since the particles are indistinguishable, this group should be viewed as a "gauge" symmetry of the system, and states transforming in the same representation have to be identified. Moreover, since all physical operators are required to commute with the permutation group, each irreducible component is a superselection sector. Therefore, one can project the Hilbert space to only some of the irreps of $S_{N}$. Further, only one state in each irrep need be kept as a representative of the multiplet of physically equivalent states. The resulting reduced space constitutes a consistent quantization of $N$ indistinguishable particles. The choice of included irreps constitutes a 
choice of quantum statistics. In particular, parabosons correspond to including only irreps with up to $p$ rows in their Young tableau, while parafermions to ones with up to $p$ columns. Clearly the cases $p=1$ reduce to ordinary fermions and bosons.

This description relies on a canonical quantization of the many-body system. It is of interest to also have a path-integral formulation of a quantum system, since this complements and completes the conceptual framework and usually offers orthogonal intuition in several cases. For ordinary statistics this question was studied by Laidlaw and DeWitt [15]. In this paper, we provide such a realization for parastatistics, or, in general, for any statistics where the Hilbert space is embedded in the tensor product of $N$ one-particle Hilbert spaces (note that this excludes anyons and braid statistics).

The starting point will be the coordinate representation of the full (unprojected) Hilbert space, spanned by the position eigenstates $\left.\left|x_{1}, \ldots x_{N}>\equiv\right| x\right\rangle$ (where $x_{i}$ can be in a space of any dimension). The collection of such states for a set of distinct $x_{i}$ transforms in the $N$ !-dimensional defining representation of $S_{N}$

$$
P|x>\equiv| P x>=\mid x_{P^{-1}(1)}, \ldots x_{P^{-1}(N)}>
$$

where $P$ is a permutation (the appearance of $P^{-1}$ in the above is necessary so that products of permutations be represented in the right order). If any of the coordinates $x_{i}$ coincide the above is not the full defining representation any more. The set of such states, however, is of measure zero (the coordinate space assumed continuous) and thus they can be safely ignored. (We assume that there are no interactions singular at coincidence points that might dynamically make such states of nonzero weight.)

Projecting the Hilbert space to an irrep $R$ of $S_{N}$ amounts to keeping only linear combinations of states within this multiplet transforming in $R$, that is,

$$
\left|a ; x>=\sum_{P} C_{a}(P) P\right| x>, \quad a=1, \ldots d_{R}, \quad d_{R}=\operatorname{dim}(R) .
$$


where the sum is over all elements of the permutation group and $C_{a}(P)$ are appropriately chosen coefficients. If we denote with $R_{a b}(P)$ the matrix elements of the permutation $P$ in the representations $R$,

$$
P\left|a, x>=\sum_{b} R_{a b}\left(P^{-1}\right)\right| b, x>
$$

The defining representation decomposes into irreducible components, classified by Young tableaux, each appearing with a certain multiplicity. Should we keep only one irrep out of each multiplicity or the whole multiplet? To decide it, note that if instead of the base state $\mid x>$ for the construction of the states $\mid a, x>$ we choose a different permutation $P_{o} \mid x>$, then although the new states $\mid a, P_{o} x>$ constructed through (2) still transform in the irrep $R$, in general they are not linear combinations of $\mid a, x>$ but rather span a different copy of $R$. Since we can continuously move in the configuration space from $\mid x>$ to $P_{o} \mid x>$, we conclude that we must keep all irreps $R$ within each multiplet. (In other words, although for each point in the Hilbert space $\mid x>$ this multiplet is reducible, the fiber of these representations over the Hilbert space is connected and irreducible.)

To realize explicitly the above, we construct the states

$$
\left|a b, x>=\sqrt{\frac{d_{R}}{N !}} \sum_{P} R_{a b}(P) P\right| x>
$$

Using the group property of the representation $R\left(P_{1}\right) R\left(P_{2}\right)=R\left(P_{1} P_{2}\right)$, we deduce that under the action of the group $S_{N}$ and under change of base point $x$ the above states transform as:

$$
P\left|a b, x>=\sum_{c} R_{a c}\left(P^{-1}\right)\right| c b, x>, \quad\left|a b, P x>=\sum_{c} R_{c b}\left(P^{-1}\right)\right| a c, x>
$$

Thus we see that the first index in these states labels the different elements of a single irrep $R$, while the second index labels the different equivalent irreps in the 
multiplet. Since both indices take $d_{R}$ values, we recover the standard result that each irrep of $S_{N}$ is embedded in the defining representation a number of times equal to its dimension.

Consider now the matrix element $\langle a b, x|A| c d, y\rangle$, where $A$ is any physical operator, that is, any operator commuting with all elements $P$ of $S_{N}$. Substituting the definition (4) and using the unitarity of $P\left(P^{\dagger}=P^{-1}\right)$ and of $R\left(R_{a b}^{*}(P)=\right.$ $\left.R_{b a}\left(P^{-1}\right)\right)$ we obtain, after a change in summation variable,

$$
<a b, x|A| c d, y>=\frac{d_{R}}{N !} \sum_{P, P^{\prime}, e} R_{b e}\left(P^{\prime}\right) R_{e a}\left(P^{-1}\right) R_{c d}(P)<x\left|A P^{\prime}\right| y>
$$

Using further the orthogonality (Shur's) relation (see, e.g., [16])

$$
\sum_{P} R_{a b}(P) R_{c d}\left(P^{-1}\right)=\frac{N !}{d_{R}} \delta_{a d} \delta_{b c}
$$

we finally obtain

$$
<a b, x|A| c d, y>=\sum_{P} \delta_{a c} R_{b d}(P)<x|A| P y>
$$

Let us first choose $A=1$. Then the above provides the overlap between the states

$$
<a b, x \mid c d, y>=\sum_{P} \delta_{a c} R_{b d}(P) \delta(x-P y)
$$

For $x$ in the neighborhood of $y$ it is $P=1$ which contributes to the normalization, for which $R_{b d}(1)=\delta_{b d}$ and we recover the standard continuous normalization between the states.

Now we can choose $A=e^{-i H t}$, where $H$ is the hamiltonian, and thus find the propagator $G(a b, x ; c d, y \mid t)$ between the states of the system. It is clear from (8) that the first index $a$ in the state $\mid a b, x>$ propagates trivially. Since this is the index that corresponds to the different but physically equivalent states within each irrep 
$R$, we conclude that the required projection of the Hilbert space to the physical subspace amounts to simply omitting this index from all states. (That is, freeze this index to the same fixed value for all states of the theory; no physical quantity will ever depend on the choice of this value.) On the other hand, the second index, corresponding to different equivalent irreps, does not propagate trivially and must, as argued before, be kept. We are led therefore to the physical states $|b a, x>\rightarrow| a, x>$ and the propagator

$$
G_{R}(a, x ; b, y \mid t)=\sum_{P} R_{a b}(P) G(x, P y ; t)
$$

where $G(x, P y ; t)=<x\left|e^{-i H t} P\right| y>$ is the usual many-body propagator. We note that, due to the transformation property (5), the states $\mid a, P x>$ are linear combinations of states $\mid a, x>$. Therefore, projecting down to the physical subspace corresponding to $R$ amounts to trading the original $N$ ! copies of physically equivalent states $\mid P x>$ for a number $d_{R}$ of global internal degrees of freedom for the system, labeled by the index $a$.

It is now easy to write down the path integral corresponding to identical particles quantized in the $R$-irrep of $S_{N}$. $G(x, P y ; t)$ can be expressed as an $N$-body path integral in the standard way, with particles starting from positions $x_{i}$ and ending in positions $P y_{i}=y_{P^{-1}(i)}$. Since all permutations of particle positions are physically equivalent, (10) instructs us to sum over all sectors where particles end up in such permuted positions, weighted with the factors $R_{a b}(P)$ depending on the internal degrees of freedom of the initial and final states. From (4), (9) we can write the completeness relation within the physical subspace

$$
I_{R}=\int \frac{d^{N} x}{N !} \sum_{a}|a, x><a, x|
$$

and with the use of (11) it is easy to prove that the above path integral is unitary, 
that is,

$$
\int \frac{d^{N} y}{N !} \sum_{b} G(a, x ; b, y \mid t) G\left(b, y ; c, z \mid t^{\prime}\right)=G\left(a, x ; c, z \mid t+t^{\prime}\right)
$$

The extension to parabosons, parafermions or any similar statistics is immediate. Let $S=\left\{R_{1}, \ldots R_{n}\right\}$ be the set of allowed irreps of $S_{N}$ in the Hilbert space. The internal degree of freedom now takes values $A=(R, a)$, where $R \in S$ and $a=1, \ldots d_{R}$ labels the internal degrees of freedom within each irrep. So, overall, $A$ takes $d_{R_{1}}+\cdots d_{R_{n}}$ different values. The propagator (and corresponding path integral) is obviously

$$
G_{S}(A, x ; B, y \mid t)=\sum_{P} S(P)_{A B} G(x, P y ; t), \quad \text { where } S(P)_{A B}=\delta_{R_{A}, R_{B}}\left(R_{A}\right)_{a b}(P)
$$

For parabosons (parafermions) of order $p, S$ is the set of Young tableaux with up to $p$ rows (columns). We note that the irreps for parafermions are the duals of those for parabosons (the dual of a tableau is the tableau with rows and columns interchanged). In an appropriate basis, the representation matrices of dual irreps $R, \tilde{R}$ are real and satisfy

$$
\tilde{R}_{a b}(P)=(-1)^{P} R_{a b}(P)
$$

where $(-1)^{P}$ is the parity of the permutation. We arrive then at the relation between the weights for parabosons and parafermions of order $p$ :

$$
S_{p F}(P)_{A B}=(-1)^{P} S_{p B}(P)_{A B}
$$

This extends a similar relation for ordinary fermions and bosons, for which there are no internal degrees of freedom and $S_{B}(P)=1$.

From the path integral we can evaluate the partition function, by simply shifting to the euclidean periodic propagator $G_{E}(\beta)=e^{-\beta H}$ and summing over all 
initial and final states, with the measure implied by (11). Given that

$$
\sum_{a} R_{a a}(P)=\operatorname{tr} R(P)=\chi_{R}(P)
$$

we get the expression in terms of the characters of $S_{N}$

$$
Z_{S}(T)=\int \frac{d^{N} x}{N !} \sum_{P} S(P)<x\left|G_{E}(\beta)\right| P x>, \text { where } S(P)=\sum_{R \in S} \chi_{R}(P)
$$

The interpretation in terms of a periodic euclidean path integral is obvious. The characters $\chi_{R}(P)$ are a set of integers, and thus the "statistical factors" $S(P)$ weighing each topological sector of the path integral are (positive or negative) integers. In the case of parabosons of any order $p$, however, we note that the statistical weights are positive (or zero) integers. The ones for parafermions can be either positive or negative, as given by

$$
S_{p F}(P)=(-1)^{P} S_{p B}(P), \quad S_{p B}(P) \geq 0
$$

We do not have a general formula for $S_{p B}(P)$ for arbitrary $p$.

From the above results we can derive the partition function for a gas of parastatistical particles as well as the allowed occupancy of single-particle states. Consider a collection of non-interacting particles, for which the hamiltonian is separable into a sum of one-body hamiltonians $H=\sum_{i} H\left(x_{i}\right)$. Let the energy eigenvalues of the one-body problem be $\epsilon_{i}$ and the corresponding one-body Boltzmann factors $z_{i}=e^{-\beta \epsilon_{i}}$. Consider now a sector of the euclidean path integral characterized by the permutation of final points $P$. It is clear that this path integral $Z_{P}$ decomposes into a product of disconnected components, characterized by the fact that the particle worldlines in each component mix particles only within the same component. This means that, within each component, particles mix under a cyclic permutation (since following the worldline of each particle must successively lead to every other 
particle in the component). Each element $P$ of $S_{N}$ is then decomposed into a product of commuting cyclic permutations. The number of particles $n$ participating in each cyclic permutation constitute the cycles of $P$ (obviously $\sum n=N$ ). We are led, thus, to the fact that for noninteracting particles

$$
Z_{P}=\prod_{n \in \operatorname{cycles}(P)} Z_{n}
$$

The path integral $Z_{n}$ for a cyclic permutation of $n$ particles, on the other hand, can be thought of as the path integral of a single particle winding $n$ times around euclidean time $\beta$. This means that

$$
Z_{n}(\beta)=Z_{1}(n \beta)=\sum_{i} z_{i}^{n} \equiv W_{n}\left[z_{i}\right]
$$

and the corresponding expression for $Z_{P}$ becomes

$$
Z_{P}=\prod_{n \in \operatorname{cycles}(P)} W_{n}\left[z_{i}\right]
$$

The expression for the full partition function then becomes

$$
Z_{S}=\sum_{R \in S} \sum_{P} \frac{1}{N !} \chi_{R}(P) \prod_{n \in \operatorname{cycles}(P)} W_{n}\left[z_{i}\right]
$$

We recognize the sum over $P$ in (22) as Frobenius' relation, connecting the sum over the Schur functions $W_{n}\left[z_{i}\right]$ to the characters of $S U(M) \chi_{R}\left[z_{i}\right]$. We get the final result

$$
Z_{S}=\sum_{R \in S} \chi_{R}\left[z_{i}\right]=\sum_{R \in S} \frac{\operatorname{det}\left(z_{i}^{N-1-j+\ell_{j}}\right)}{\operatorname{det}\left(z_{i}^{N-1-j}\right)}
$$

where $\ell_{j}$ is the length of the $j$-th row of the Young tableau of $R$. This reproduces the result of Chaturvedi for the partition function [17] and an earlier result of Suranyi for $p=2$ [18]. We stress that the above result holds only for noninteracting 
particles. For interacting particles the topologically disconnected components $Z_{n}$ of the path integral $Z_{P}$ are still dynamically connected and factorization fails. One has to go back to the full expression (17) for the partition function in that case.

To find the degeneracy of states, we need to decompose $\chi_{R}\left[z_{i}\right]$ appearing in (23) in monomials $\prod_{i} z_{i}^{p_{i}}$

$$
\sum_{R \in S} \chi_{R}\left[z_{i}\right]=\sum_{\left\{p_{i}\right\}} D\left[p_{i}\right] \prod_{i} z_{i}^{p_{i}}
$$

The coefficients $D\left[p_{i}\right]$ of these monomials, called Kostka-Foulkes numbers [17, 19], are non-negative integers which determine the degeneracy of the state with $p_{i}$ particles occupying each energy level $\epsilon_{i}$. To find these integers in a systematic way, we use the following trick: consider that the particles are bosons and have an internal degree of freedom transforming in the fundamental of $S U(N)$ (in fact, $S U(M)$ with $M \geq N$ would also do). Since under total permutation of particle coordinates and internal degrees of freedom the states must transform trivially, we conclude that the irrep of the color $S U(N)$ for each state must be the same as the irrep of the coordinate permutation group $S_{N}$ (meaning they have the same Young tableau and thus the same symmetries). A state with $p$ particles in the same level $\epsilon_{i}$ transforms in the $p$-fold symmetric irrep of $S U(N)$. Therefore, a state with occupancies $p_{i}$ for each level transforms under the direct product of $p_{i}$-fold symmetric irreps, one for each level $\epsilon_{i}$. Decomposing this product into irreducible components, we will obtain each representation $R$ of $S U(N)$ a number of times $D_{R}\left[p_{i}\right]$. Each such irrep will transform under a similar irrep of $S_{N}$ and thus will correspond to a unique physical state in the quantization of $N$ identical particles in the representation $R$. Therefore, the degeneracy $D\left[p_{i}\right]$ can be found by summing the number of times that each allowed irrep $R \in S$ appears in the direct product of symmetric irreps $p_{i}$, which can be found using standard $S U(N)$ Young tableaux composition rules. We also see that, if the internal degree of freedom group is chosen to be $S U(p)$ (where $p$ may be smaller that $N$ ), we will only get irreps with up to $p$ rows. Therefore, we recover the known result that parabosons of order $p$ 
can be viewed as bosons with an internal $S U(p)$ symmetry, where we identify each irrep of $S U(p)$ as a unique physical state [11,12]. A similar construction can be repeated starting from fermions instead of bosons. We recover a dual expression for the degeneracies $D\left[p_{i}\right]$, where now we form the direct product of $p_{i}$-fold fully antisymmetric irreps of $S U(N)$, and a similar expression of parafermions of order $p$ as fermions with an $S U(p)$ internal symmetry.

As was argued in $[10,14]$, parastatistics particles obey the cluster decomposition principle, in the sense that the density matrix obtained by tracing over a subset of particles which decouple from the system can be constructed as a possible density matrix of the reduced system of remaining particles. From (17), however, we see that the partition function of two dynamically isolated sets of particles $N_{1}$ and $N_{2}$ does not factorize into the product of the two partition functions, since the statistical weights $S(P)$ in general do not factorize into $S\left(P_{1}\right) S\left(P_{2}\right)$ when $P$ is the product of two commuting elements $P_{1}$ and $P_{2}$. Equivalently, this means that the occupation degeneracy $D\left[p_{i}\right]$ does not factorize into the product of individual occupation degeneracies for each level $\epsilon_{i}$. This has important physical implications. If the two sets of particles are totally isolated, it does not make sense to evaluate the partition function of the total system, since the statistical distribution can never relax to the one predicted by that partition function. The individual partition functions of the subsystems are the relevant ones. If, however, the two sets are only weakly coupled, then initially each set will distribute according to its reduced partition function, but after some relaxation time (depending on the strength of the coupling between the two sets) they will relax to the joint distribution function, which, we stress, will not even approximately equal the product of the individual ones. Thus, cluster decomposition holds in an absolute sense but fails in a more realistic sense. In contrast, fermions and bosons respect cluster decomposition in both senses.

The obvious generalization of quantum statistics, based always on the assumption that the many-body Hilbert state is embedded into the tensor product of many one-body Hilbert spaces, is to generalize the set of allowed irreps $S$ beyond 
the one relevant to parastatistics. We may, however, further include more than one state for each included irrep of $S_{N}$. This seems unmotivated, in view of the fact that such states are physically indistinct, but it is certainly consistent. It could mean, for instance, that the particles have some hidden internal degrees of freedom accounting for the extra degeneracy, which are invisible to the present hamiltonian but may become dynamically relevant later. The most general situation, then, is that we include $C_{R}$ states from each irrep $R$. The generalization of all previous formulae for this case is quite immediate, $S(P)$ and $C_{R}$ being related by

$$
S(P)=\sum_{R} C_{R} \chi_{R}(P), \quad C_{R}=\frac{1}{N !} \sum_{P} S(P) \chi_{R}(P)
$$

The case of distinguishable particles ("infinite statistics" [12,20,21]), in particular, is reproduced by accepting all states in each irrep, that is, $C_{R}=d_{R}$. Since $R$ appears exactly $d_{R}$ times in the defining representation of $S_{N}, S(P)$ above becomes the trace of $P$ in that representation. But all $P \neq 1$ are off-diagonal in the defining representation, so we get $S_{i n f}(P)=N ! \delta_{P, 1}$, recovering the standard distinguishable particles result.

We summarize here by pointing out that the most general statistics of the type examined here is parametrized by any of three possible sets of numbers. The first is, as just stated, the number of states $C_{R}$ accepted for each irrep $R$ of $S_{N}$. Since the irreps of $S_{N}$ are parametrized by the partitions of $N$ (lengths of rows or the Young tableau), there are as many $C_{R}$ as there are partitions of $N$. The second set is the statistical weights $S(P)$ appearing in the partition function (euclidean path integral). Clearly these weights are invariant under conjugation of $P \rightarrow Q P Q^{-1}$, since this simply amounts to a relabeling of the particle worldlines. Thus $S(P)$ depends only on the conjugacy class of $P$, that is, the cycles of $P$. The possible sets of cycles are the same as the partitions of $N$; so, again, the $S(P)$ are numbered by partitions of $N$. Finally, we could use the degeneracy of a many-body occupancy state $D\left[p_{i}\right]$ as our definition. There are as many ways to distribute particles in one-body states as there are partitions of $N$, so this set also has the same number of elements as the previous two. 
What are the restrictions or criteria to be imposed on the above parameters? The first one is unitarity, that is, the existence of a well-defined Hilbert space with positive metric. This requires that $C_{R}$ be non-negative (no negative norm states) integers (no "fractional dimension" states). The other will be what we call "strong cluster decomposition principle," that the partition function of isolated systems factorize. This is a physical criterion, rather than a consistency requirement. To summarize:

- Unitarity : $C_{R}$ non - negative integers

- Strong cluster decomposition : $S(P)=\prod_{n \in \operatorname{cycles}(P)} S(n)$ or $D\left[p_{i}\right]=\prod_{i} D\left(p_{i}\right)$

The strong cluster decomposition, in particular, implies the existence of a grand partition function, obtained (in the case of noninteracting particles) by exponentiating the sum of all connected path integrals $(P$ a cyclic permutation of degree $n)$ with weights $S(n) / n(1 / n$ is the symmetry factor of this path integral, corresponding to cyclic relabelings of the particles). The grand partition function will further factorize into a product of partition functions for each level $\epsilon_{i}$. Thus, $S(n)$ are cluster coefficients connected to $D(n)$ in the standard way

$$
\sum_{p=0}^{\infty} D(p) z^{p}=\exp \left(\sum_{n=1}^{\infty} \frac{S(n)}{n} z^{n}\right)
$$

The above formula, in fact, provides the easiest way to relate $D\left[p_{i}\right]$ and $S(P)$ in the general case (no strong cluster property): simply expand the right-hand side of (26) in powers of $z$ and substitute every term $S\left(n_{1}\right) \cdots S\left(n_{k}\right)$ with $S\left(n_{1}, \ldots n_{k}\right)$. This gives $D(p)$. To find $D\left[p_{i}\right]=D\left(p_{1}, \ldots p_{k}\right)$ simply evaluate $D\left(p_{1}\right) \cdots D\left(p_{k}\right)$ using the above formula and again consolidate each product $S\left(n_{1}\right) \cdots S\left(n_{k}\right)$ into a single $S\left(n_{1}, \ldots n_{k}\right)$.

If we assume that $S(1)=D(1)=1$, then it is easy to verify that the only solution of the above two criteria is ordinary fermions and bosons. The situation is different, however, when $S(1)=D(1)=q>1$ (this would mean, e.g., that the 
particles come a priori in $q$ different "flavors"). The possibilities are manifold. All these generalized statistics share the following generic features:

- The degeneracy of the state where $n$ particles occupy different levels is $q^{n}$. (Indeed, $D(1,1, \ldots 1)=D(1)^{n}=q^{n}$.)

- If state $A$ can be obtained from state $B$ by 'lumping' together particles that previously occupied different levels, then $D(A) \leq D(B)$. (E.g., $D(3) \leq D(2,1) \leq$ $D(1,1,1)$.)

This second property is actually related to the (weak) cluster decomposition as formulated in [14], which is obviously covered by the strong cluster property.

The above possibilities include the obvious special cases of $q_{1}$ bosonic flavors and $q_{2}$ fermionic ones $\left(q_{1}+q_{2}=q\right)$, for which $S(n)=q_{1}-(-1)^{n} q_{2}$, along with many other. As an example, we give the first few degeneracies for many-particle level occupation for all statistics with $q=2$ :

$$
\begin{aligned}
& D(1)=2, D(2)=4, D(3)=8 \\
& D(1)=2, D(2)=3, D(3)=6,5,4(B+B) \\
& D(1)=2, D(2)=2, D(3)=4,3,2(B+F), 1,0 \\
& D(1)=2, D(2)=1, D(3)=2,1,0(F+F) \\
& D(1)=2, D(2)=0, D(3)=0
\end{aligned}
$$

The specific choices denoted by $B+B, B+F$ and $F+F$ are the ones corresponding to two bosonic, one bosonic and one fermionic, and two fermionic flavors respectively. The topmost statistics could be termed "superbosons" and the bottom one "superfermions" of order 2. We also remark here that the " $(p, q)$ statistics" introduced in [14] can be realized as particles with $p$ bosonic and $q$ fermionic flavors, where we identify each multiplet transforming irreducibly under the supergroup $S U(p, q)$ as a unique physical state.

Finally, we direct our attention to the first known attempt to generalize the ordinary Fermi or Bose statistical mechanics, by Gentile [22]. The rule is simply 
that up to $p$ particles can be put in each single-particle level. This corresponds to $D(n)=1$ for $n \leq p$, and $D(n)=0$ otherwise. This has been criticized [7] on the grounds that fixing the allowed occupations for each single-particle state is not a statement invariant under change of single-particle basis. It is clear that, in the language of this paper, any statistics satisfying the unitarity requirement is consistent and basis-independent. Therefore, Gentile statistics must violate unitarity. Indeed, it is easy to check that all weights $C_{R}$ for such statistics are integers (this is generic for all statistics with integer $D(n)$ ), but not necessarily positive. In the specific case of $p=2$, e.g., where up to double occupancy of each level is allowed, the degeneracies of each irrep of $S_{N}$ (parametrized, as usual, by the length of Young tableau rows) up to $N=5$ are

$$
C_{2}=C_{21}=C_{22}=C_{221}=1, C_{111}=C_{1111}=C_{2111}=-1, \text { else } C_{R}=0
$$

We see that representations 111, 1111, 2111 correspond to ghost (negative norm) states and their effect is to subtract (rather than add) degrees of freedom. We also remark that the path integral realization of exclusion statistics exhibits both negative and fractional statistical weights, signaling breakdown of unitarity [23]. This is inconsequential in that case, since exclusion statistics is valid only as a macroscopic (statistical) description of some (interacting) systems of particles.

In conclusion, we have presented the many-body propagators and corresponding path integrals of particles obeying parastatistics or any other type of statistics based on irreps of the permutation group. We argued that there are many possible unitary generalizations obeying the strong cluster decomposition principle, although they all require more than one flavor of particles. Several other directions of investigation and open questions suggest themselves. To name a few, the statistical mechanics of such generalized statistics particles should be examined. Also, it should be checked if they can be realized as particles with specific hidden internal symmetries and an appropriate projection of the Hilbert space, in a fashion similar to parastatistics. Independently, it would be interesting to see if 
Gentile statistics can be consistently realized by introducing 'benign' ghosts which account for the negative norm states while decoupling from all physical processes, just as in gauge theories. Finally, a similar analysis could be attempted for generalized particle statistics in $2+1$ dimensions. In fact, a similar treatment, based on the permutation group, has been used to obtain perturbative results for anyonic

particles [24-26]. It would be interesting to examine whether non-abelian irreps of the braid group, instead of the permutation group, could be considered.

Acknowledgements: I would like to thank J. Myrheim for discussions.

\section{REFERENCES}

1. J.M. Leinaas and J. Myrheim, Nuovo Cimento 37B, 1 (1977).

2. G.A. Goldin, R. Menikoff and D.H. Sharp, J. Math. Phys. 21, 650 (1980); 22, 1664 (1981); Phys. Rev. D28, 830 (1983).

3. F. Wilczek, Phys. Rev. Lett. 48, 1114 (1982); 49, 957 (1982).

4. Y.S. Wu, Phys. Rev. Lett. 52, 2103 (1984).

5. F.D.M. Haldane, Phys. Rev. Lett. 67 (1991) 937.

6. J. Myrheim, Anyons (Notes for the Course on Geometric Phases, ICTP, Trieste 6-17 Sept. 1993).

7. O.W. Greenberg et al., hep-ph/9306225.

8. H.S. Green, Phys. Rev. 90, 270 (1953).

9. O. Steinmann, Nuovo Cimento 44, A755 (1966).

10. P.V. Landshoff and H.P. Stapp, Ann. of Phys. 45, 72 (1967).

11. Y. Ohnuki and S. Kamefuchi, Phys. Rev. 170, 1279 (1968); Ann. of Phys. 51, 337 (1969).

12. S. Doplicher, R. Haag and J. Roberts, Comm. Math. Phys. 23, 199 (1971); 35, 49 (1974). 
13. A.M.L. Messiah and O.W. Greenberg, Phys. Rev. B136, 248 (1964); B138, 1155 (1965).

14. J.B. Hartle and J.R. Taylor, Phys. Rev. 178, 2043 (1969); R.H. Stolt and J.R. Taylor, Phys. Rev. D1, 2226 (1970); Nucl. Phys. B19, 1 (1970); J.B. Hartle, R.H. Stolt and J.C. Taylor, Phys. Rev. D2, 1759 (1970).

15. M.G.G. Laidlaw and C.M. DeWitt, Phys. Rev. D3, 1375 (1971).

16. M. Hammermesh, Group Theory, Addison-Wesley Eds. (1962).

17. S. Chaturvedi, U. Hyderabad preprint, hepth/9509150.

18. P. Suranyi, Phys. Rev. Lett. 65, 2329 (1990).

19. C. Kostka, Crelles Journal 93, 98 (1882); H.O. Foulkes, Permutations, Eds. Gauthier-Villars, Paris (1974).

20. A.B. Govorkov, Theor. Math. Phys. 54, 234 (1983).

21. O.W. Greenberg, Phys. Rev. Lett. 64, 705 (1990).

22. G. Gentile, Nuovo Cimento 17, 493 (1940).

23. A.P. Polychronakos, Phys. Lett. B365, 202 (1996).

24. J. Myrheim and K. Olaussen, Phys. Lett. B299, 267 (1993).

25. J. Desbois, C. Heinemann and S. Ouvry, Phys. Rev. D51, 942 (1995).

26. A.D. de Veigy, Nucl. Phys. B458 [FS], 533, 1996). 